\title{
Identification and Discrimination of the Limb Motions using Brain Waves from Motor Imagery
}

\author{
Minoru Sasaki ${ }^{*}$, Toshifumi Kojima ${ }^{1}$, Kojiro Matsushita ${ }^{1}$, Satoshi Ito ${ }^{1,}$ Titus Mulembo ${ }^{1,}$ Muhammad Ilhamdi Rusydi ${ }^{2}$ \\ ${ }^{1}$ Department of Mechanical Engineering, Gifu University, 1-1 Yanagido, Gifu, 501-1193, Japan \\ ${ }^{2}$ Department of Electrical Engineering, Andalas University, Limau Manis, Padang City, 25163, Indonesia \\ ${ }^{*}$ Corresponding author
}

\begin{abstract}
This research identified and distinguished limb motions by the use of Neural Networks (NN), Support Vector Machines (SVM) and the Electro-encephalogram (EEG). EEG enabled motor imagery of the limbs. By comparing the EEG power at rest with that of the limb's motor imagery, measurements from the electrodes and EEG frequency bands were examined. In this study, EEG with a limited frequency band (from $25 \mathrm{~Hz}$ to $30 \mathrm{~Hz}$ ) obtained from the electrodes $\mathrm{C} 3, \mathrm{C} 4, \mathrm{P} 3$ and $P 4$ was used. The features were extracted by the use of Fast Fourier Transform (FFT). The results showed that three out of four subjects were able to identify and distinguish their limb motions at a success rate of over $70 \%$. Furthermore, the rates of identification and discrimination of the limb motions were slightly higher for the Support Vector Machines than for the Neural Networks.
\end{abstract}

Keywords- Electro-encephalogram (EEG); Brain Computer Interface (BCI); motor imagery; limbs motion; Fast Fourier Transform (FFT); Neural Network (NN); Support Vector Machine (SVM)

\section{INTRODUCTION}

People with severe disabilities in motor functions such as amyotrophic lateral sclerosis (ALS) need adequate nursing care. In recent years, research on brain computer interface (BCI) was conducted as a method for providing such people with support for living and communication with other people. The BCI system detects the user's intentions from the brain waves and uses it to control external devices.

Previous studies on BCI included mental task classification [1], motion estimation based on exercise images [2-4], and cognitive state discrimination [5]. Some studies focus on use of EEG at the time of motion image, performing of brain wave analysis of left and right hands and both feet [6]. Also the discrimination of the right and the left elbow motion images [2], etc. were also performed; there are only few studies which perform the discrimination task. Previous research in our laboratory estimated the limb motion using the $\mathrm{NN}$ or the SVM. Note that these studies used brain waves for moving the body. However, they were not considered as brain waves at the time of imaging.

This research aims at realizing a new BCI system by distinguishing four movements from the brain waves obtained by imaging limb movements by use of NN and SVM. As a feature of this research, frequency bands of the electrodes and the brain waves which were specifically related to motion image processing during the pre-processing were investigated. The comparison of the change of the image intensity of the EEG both at the rest and during the operation time was performed. There are various methods of signal processing, such as frequency spectral analysis and independent component analysis. Both methods reported that the value of the power spectrum changes before and after the image [8]. In this study, the time window Fast Fourier Transform (FFT) was performed while shifting the power spectrum little by little. The change in the amount of the power spectrum was used as a feature amount. Note that NN and SVM are often used in classification of EEG signals because it is often possible to obtain good solutions to multidimensional quantity data. They are also applied on problems that cannot be linearly separated.

\section{MEASUREMENT OF EEG}

\section{A. Biomedical Signal Detection Device}

Kansei Spectral Analysis System obtained from Brain Function Laboratory, Inc was used for the measurement of brain waves. First, brain waves were measured from a pasteless electrode helmet. Next, the measured input signal was amplified and analog to digitally conversion done by the EEG (Electroencephalogram) head box. Later on it was digitally filtered by a processor box. Finally, the digital data was transferred to the computer where the brain waves were displayed.

\section{B. Measurement Points}

For the brain wave detection, the right earlobe reference monopole guidance where right earlobe was the reference electrode was performed [9]. The electrodes arrangement on the scalp was based on the internationally defined "10-20 law" where 14 sites were used [10]. The electrode arrangement was as shown in Figure 1. 


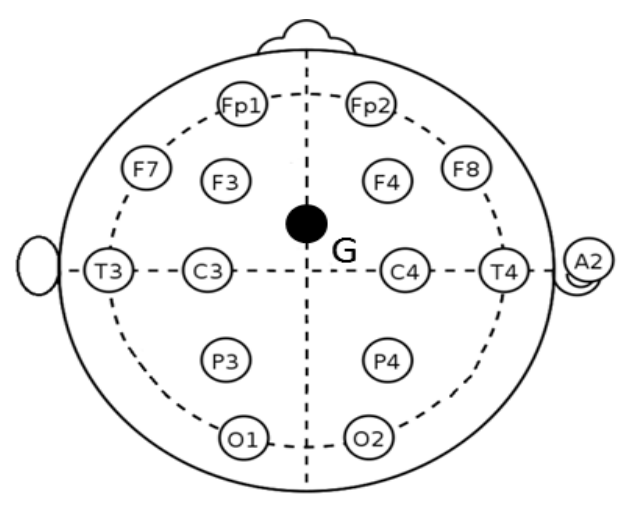

FIGURE I. THE 10-20 POSITION OF ELECTRODES.

TABLE I. BIO DATA OF ALL THE SUBJECTS.

\begin{tabular}{|c|c|c|c|}
\hline & Age & Sex & Handedness \\
\hline Subject 1 & 23 & Man & Right \\
\hline Subject 2 & 21 & Man & Right \\
\hline Subject 3 & 21 & Man & Left \\
\hline Subject 4 & 22 & Man & Right \\
\hline
\end{tabular}

\section{Measurement Method}

Four healthy volunteers were instructed to perform the ascending images of the right hand, the left hand, the right foot and the left foot while their eyes were closed. The measurement duration for one motion was 5 seconds. I signaled 1 second before the start of measurement and asked the volunteers to start the imaging. After that, they maintained the rest state until the next signal. The above measurement was just one set. The measurement for each operation was performed for 30 sets per subject and a total of 120 data was measured. Finally, we measured 30 sets brain waves at rest (this is when there was no motion image). The sampling frequency used was $200 \mathrm{~Hz}$. Table 1 shows the bio data of the four subjects.

\section{Measurement Result}

The brain waves of the four subjects at the time of the motion image and at rest were compared and there was no difference in change in voltage value. Changes in the brain waves due to motion images could not be extracted from the measured brain waves data. Thus they are not suitable as feature quantities. Therefore, in order to extract feature quantities related to motion image processing, signal processing had to be applied to the measured EEG and then examined.

\section{EXTRACTION OF FEATURES}

\section{A. Pre-processing}

During the motion imaging, the intensity of the $\beta$ wave band increases (event-related synchronization (ERS) or eventrelated desynchronization (ERD) around the cerebral somatosensory field (motor cortex) [11].

In the preprocessing, the band intensity was calculated and compared for during the time of imaging and at the rest times, respectively. The frequency bands of the electrodes and the brain waves, which are closely related to motion image processing, were examined. The band intensity was calculated by applying a bandpass filter to the measured brain wave data, squaring it, and smoothing it using the simple moving average $[6,11]$. Therefore, the frequency band of the band pass filter was limited to the $\beta$ wave band $(13-30 \mathrm{~Hz})$, and the electrodes used for calculating the band intensity around the somato-sensory field (motor field) were C3, C4, P3 and P4. changes in the intensity were compared while changing the frequency band of the filter in the $\beta$ wave band, and based on the result, the electrode to be used and the frequency band were determined.

Figure 2 shows the intensity change of subject 1 at rest when filter frequency band was set to $25-30 \mathrm{~Hz}$. The graph represents the electrodes $\mathrm{C} 3, \mathrm{C} 4, \mathrm{P} 3$ and $\mathrm{P} 4$ from the top. Figure 3, Figure 4, Figure 5 and Figure 6 show the change in intensity at each motion image at the frequency band of 25-30 $\mathrm{Hz}$.

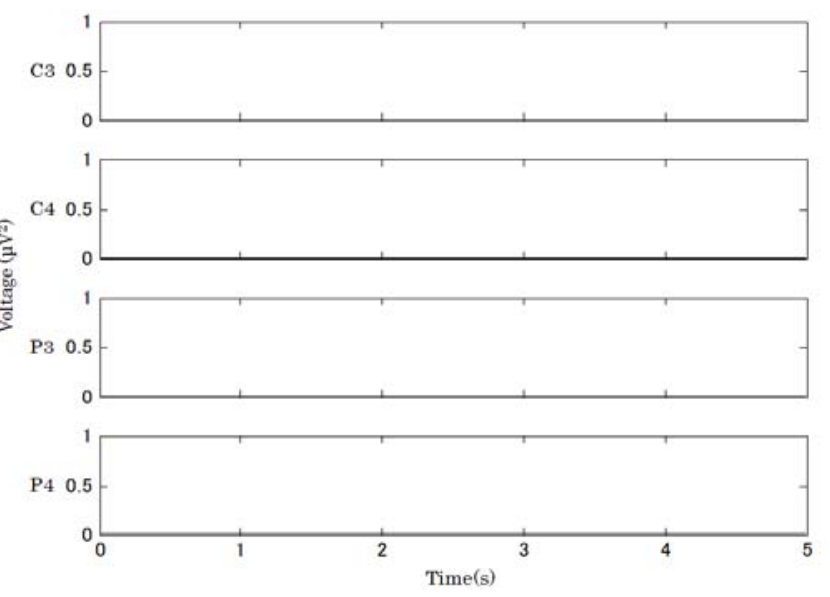

FIGURE II. ERS AT REST.

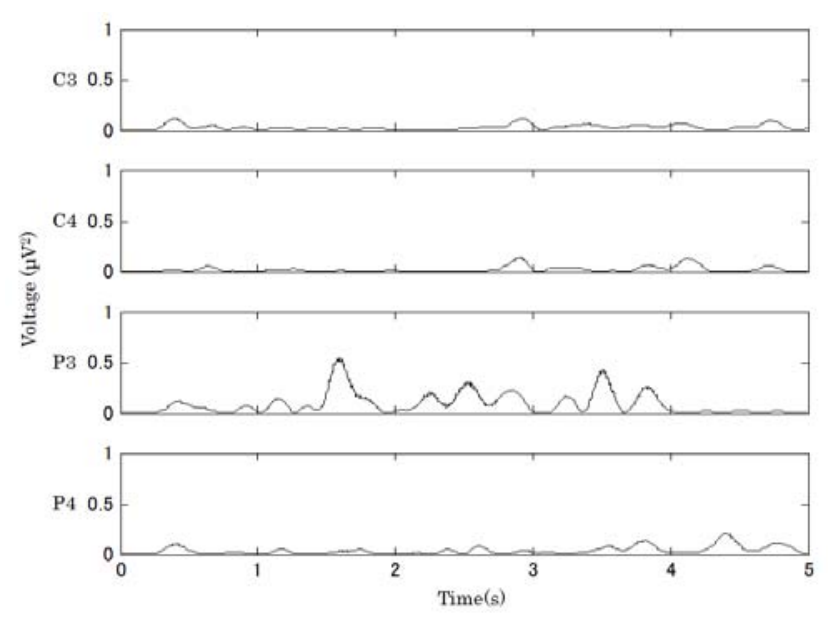

FIGURE III. ERS OF THE RIGHT HAND MOTOR IMAGERY. 

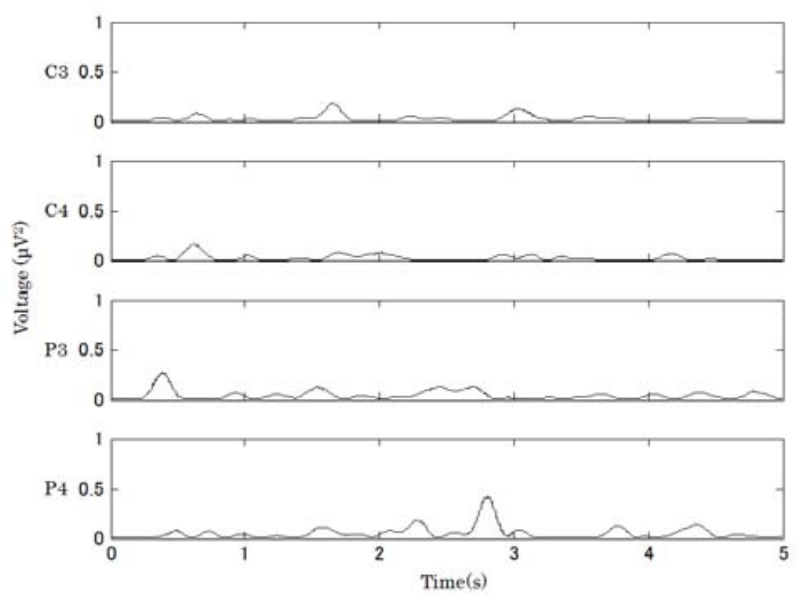

FIGURE IV. ERS OF THE LEFT HAND MOTOR IMAGERY.
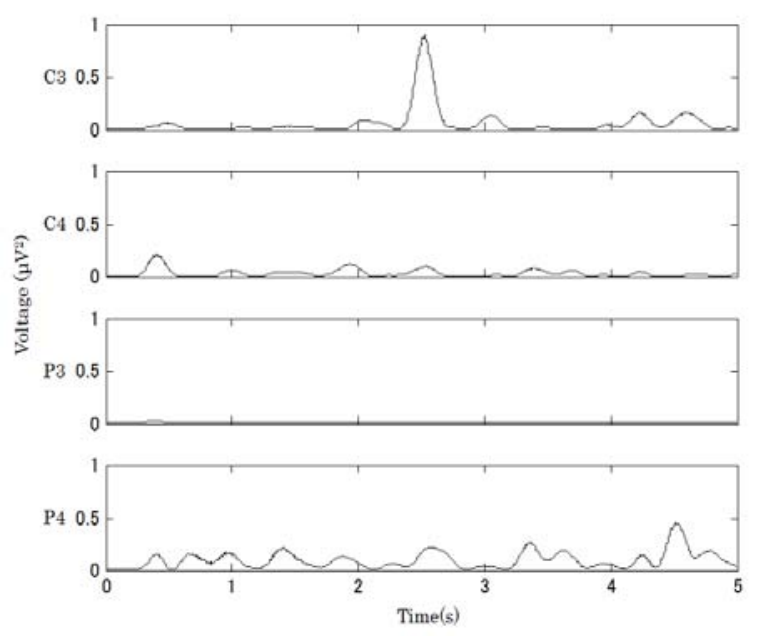

FIGURE V. ERS OF THE RIGHT LEG MOTOR IMAGERY.
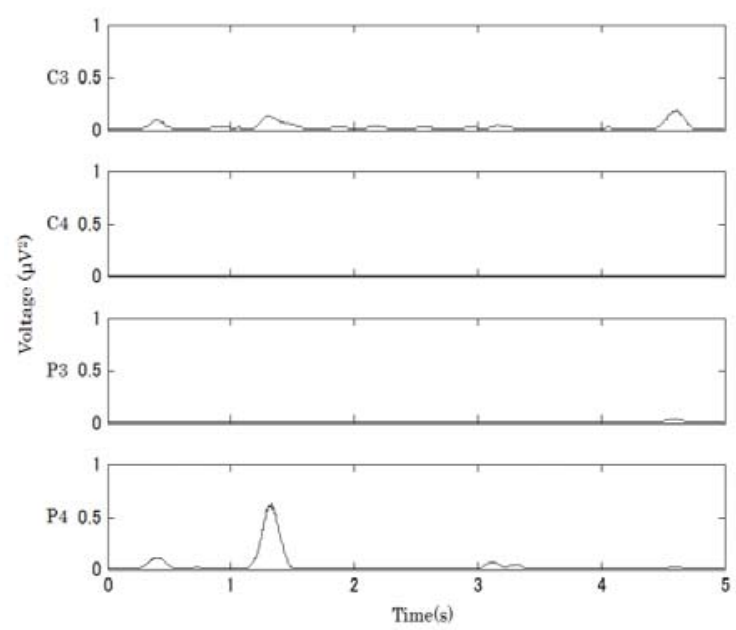

FIGURE VI. ERS OF THE LEFT LEG MOTOR IMAGERY.

From these results, when the frequency band was limited to $25-30 \mathrm{~Hz}$, there was an intensity change (ERS). Although at rest there was no change of intensity. This change is thought to be related to brain activity during image processing. Therefore, the frequency band selected was $25-30 \mathrm{~Hz}$. In addition, the electrodes where the intensity change appeared tended to be different for each operation, and therefore all the electrodes C3, C4, P3 and P4 were used.

\section{B. Features}

For the feature quantity, a bandpass filter of $25-30 \mathrm{~Hz}$ was applied to the raw data of the brain wave and the component of $25-30 \mathrm{~Hz}$ was taken out. The amount of change of the power spectrum was obtained by use of the FFT processing. Figure 7 shows the result of FFT analysis after the application of the band pass filter.

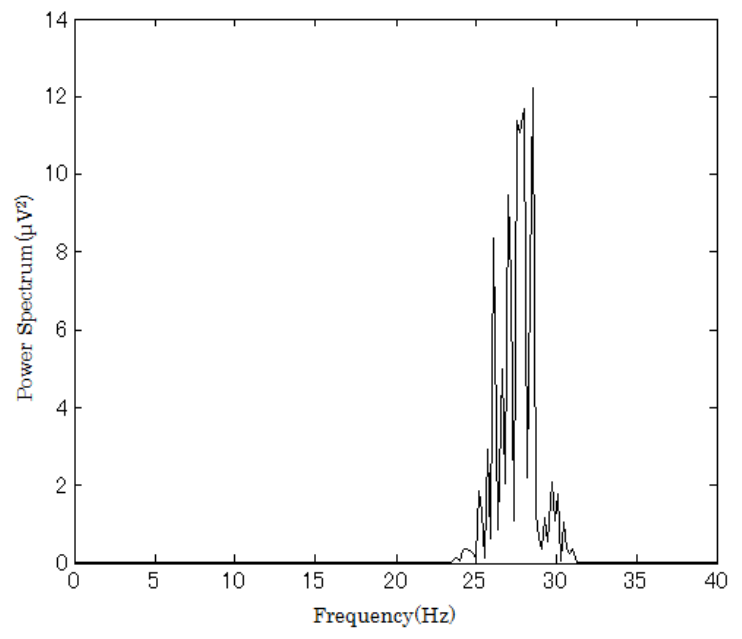

FIGURE VII. FFT ANALYSIS.
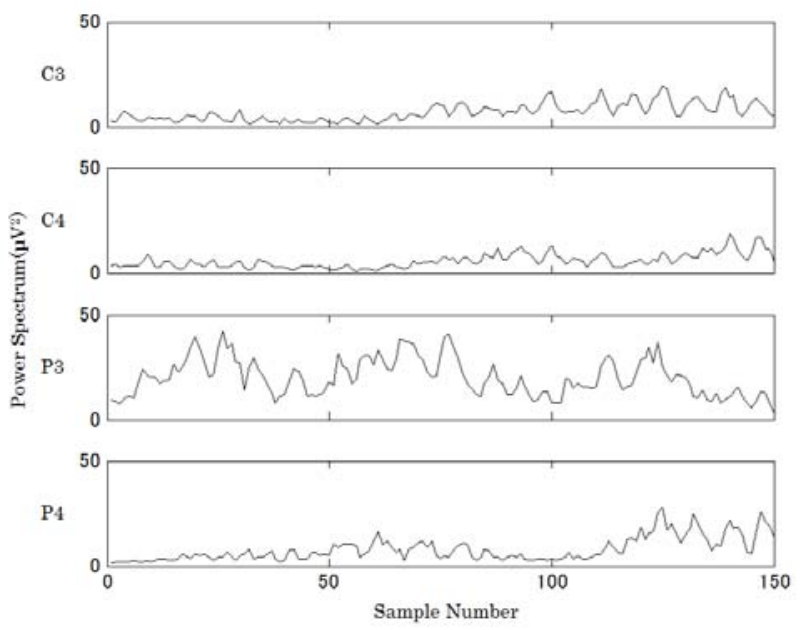

FIGURE VIII. POWER SPECTRUM OF THE RIGHT HAND MOTOR IMAGERY. 

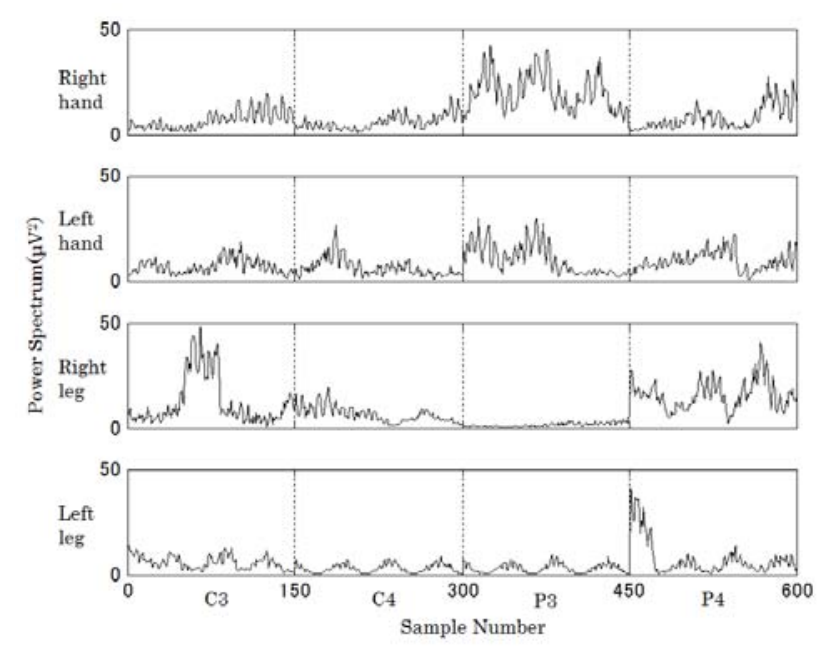

FIGURE IX. INPUT DATA.

Figure 8 shows the waveforms obtained from recording of the transition of the power spectrum when subject 1 performed the right-hand motion image. The graph shows data obtained from the electrodes C3, C4, P3, P4. From the Figure 8, the power spectrum changes significantly at $\mathrm{P} 3$.

For the discrimination data, four data sets waveforms (C 3, $\mathrm{C} 4, \mathrm{P} 3$, and $\mathrm{P} 4$ ) from subject 1 were put side to side. As shown in Figure 9. The input data was used for distinguishing between the top right hand motion image, the left hand motion image, the right foot motion image and the left foot motion image.

\section{DisCRIMINATION OF EXTREMITY MOVEMENT}

\section{A. Evaluation Method}

Learning and discrimination by use of NN and SVM were performed for each subject. 20 out of the 30 measured data of each operation were used for learning purposes and the remaining 10 were used for discrimination purposes. Furthermore, discrimination was done by changing the combinations of learning and discrimination three times, and then evaluated based on the average values [1].

\section{B. Discrimination by NN and SVM}

This study utilized the toolbox for NN of the numerical analysis software "MATLAB" developed by Math Works. A sigmoid function was used as a neuron unit of the intermediate layer and of the output layer, with the intermediate layer being a hierarchical one layer. There were 600 data for discrimination for each operation as shown in Figure 9. The number of input layers was equal to the number of samples of feature quantity ( 600 data), The number of intermediate layers was 100 . The number of input layers was set to 4 , corresponding to the 4 actions. The learning data gave a teacher vector such as $[1,0,0,0]$ for each operation. It assigned a 1 value to the output corresponding to the corresponding motion and a 0 value to the others. In the discrimination by $\mathrm{NN}$, the operation having the largest value among the four outputs was judged. The learning of NN was performed by error back propagation method. The weight updating algorithm used was steepest descent method. The learning completion condition was assumed to be when the error between the teacher data and the output became $10^{-4}[1]$.

In the discrimination by SVM, a package for SVM (e 1071) called " $R$ " was used. This is a programming language for statistical analysis. The kernel uses the RBF kernel, and the parameter gamma was 0.0017 for all the subjects. This value was determined through trial and error when considering the discrimination results of the 4 subjects. The learning data gave teacher labels of A, B, C, D for each operation. A represented the brain waves in the right-hand operation image, $B$ represented the brain wave in the left-handed operation image, $\mathrm{C}$ represented the brain wave in the right foot motion image, and $\mathrm{D}$ represented the brain wave in the left foot motion image.

TABLE II. DISTINCTION RESULTS OF SUBJECT 1.

\begin{tabular}{|c|c|c|c|c|}
\hline \multicolumn{5}{|c|}{ NN } \\
\hline Motiont & $\begin{array}{c}\text { Right } \\
\text { hand }\end{array}$ & Left hand & Right leg & $\begin{array}{c}\text { Left } \\
\text { leg }\end{array}$ \\
\hline Right hand & $23 / 30$ & $7 / 30$ & $0 / 30$ & $0 / 30$ \\
\hline Left hand & $3 / 30$ & $25 / 30$ & $0 / 30$ & $2 / 30$ \\
\hline Right leg & $0 / 30$ & $0 / 30$ & $27 / 30$ & $3 / 30$ \\
\hline Left leg & $0 / 30$ & $0 / 30$ & $1 / 30$ & $29 / 30$ \\
\hline \multicolumn{5}{|c|}{ SVM } \\
\hline Ludgment & $\begin{array}{c}\text { Right } \\
\text { hand }\end{array}$ & Left hand & Right leg & $\begin{array}{c}\text { Left } \\
\text { leg }\end{array}$ \\
\hline Motion & $18 / 30$ & $9 / 30$ & $3 / 30$ & $0 / 30$ \\
\hline Right hand & $1 / 30$ & $27 / 30$ & $2 / 30$ & $0 / 30$ \\
\hline Left hand & $0 / 30$ & $0 / 30$ & $30 / 30$ & $0 / 30$ \\
\hline Right leg & $0 / 30$ & $0 / 30$ & $1 / 30$ & $29 / 30$ \\
\hline Left leg & & & & \\
\hline
\end{tabular}

TABLE III. DISTINCTION RESULTS OF SUBJECT 2.

\begin{tabular}{|c|c|c|c|c|}
\hline \multicolumn{5}{|c|}{$\mathrm{NN}$} \\
\hline $\begin{array}{l}\text { Judgment } \\
\text { Motion }\end{array}$ & $\begin{array}{l}\text { Right } \\
\text { hand }\end{array}$ & Left hand & Right leg & $\begin{array}{l}\begin{array}{l}\text { Left } \\
\text { leg }\end{array} \\
\end{array}$ \\
\hline Right hand & $25 / 30$ & $5 / 30$ & $0 / 30$ & $0 / 30$ \\
\hline Left hand & $6 / 30$ & $19 / 30$ & $2 / 30$ & $3 / 30$ \\
\hline Right leg & $0 / 30$ & $1 / 30$ & $27 / 30$ & $2 / 30$ \\
\hline Left leg & $0 / 30$ & $0 / 30$ & $5 / 30$ & $25 / 30$ \\
\hline \multicolumn{5}{|c|}{ SVM } \\
\hline Judgment & $\begin{array}{l}\text { Right } \\
\text { hand }\end{array}$ & Left hand & Right leg & $\begin{array}{l}\text { Left } \\
\text { leg }\end{array}$ \\
\hline Right hand & $24 / 30$ & $6 / 30$ & $0 / 30$ & $0 / 30$ \\
\hline Left hand & $3 / 30$ & $23 / 30$ & $3 / 30$ & $1 / 30$ \\
\hline Right leg & $0 / 30$ & $0 / 30$ & $26 / 30$ & $4 / 30$ \\
\hline Left leg & $0 / 30$ & $2 / 30$ & $11 / 30$ & $17 / 30$ \\
\hline
\end{tabular}


TABLE IV. DISTINCTION RESULTS OF SUBJECT 3.

\begin{tabular}{|c|c|c|c|c|}
\hline \multicolumn{5}{|c|}{$\mathrm{NN}$} \\
\hline $\begin{array}{l}\text { Judgment } \\
\text { Motion }\end{array}$ & $\begin{array}{l}\text { Right } \\
\text { hand }\end{array}$ & Left hand & Right leg & $\begin{array}{l}\text { Left } \\
\text { leg }\end{array}$ \\
\hline Right hand & $17 / 30$ & $6 / 30$ & $5 / 30$ & $2 / 30$ \\
\hline Left hand & $9 / 30$ & $12 / 30$ & $1 / 30$ & $8 / 30$ \\
\hline Right leg & $9 / 30$ & $2 / 30$ & $17 / 30$ & $2 / 30$ \\
\hline Left leg & $4 / 30$ & $6 / 30$ & $5 / 30$ & $15 / 30$ \\
\hline \multicolumn{5}{|c|}{ SVM } \\
\hline Motion & $\begin{array}{l}\text { Right } \\
\text { hand }\end{array}$ & Left hand & Right leg & $\begin{array}{l}\text { Left } \\
\text { leg }\end{array}$ \\
\hline Right hand & $15 / 30$ & $10 / 30$ & $3 / 30$ & $2 / 30$ \\
\hline Left hand & $5 / 30$ & $18 / 30$ & $1 / 30$ & $6 / 30$ \\
\hline Right leg & $9 / 30$ & $1 / 30$ & $20 / 30$ & $0 / 30$ \\
\hline Left leg & $2 / 30$ & $7 / 30$ & $4 / 30$ & $17 / 30$ \\
\hline
\end{tabular}

TABLE V. DISTINCTION RESULTS OF SUBJECT 4.

\begin{tabular}{|c|c|c|c|c|}
\hline \multicolumn{5}{|c|}{ NN } \\
\hline $\begin{array}{c}\text { Motgment } \\
\text { Motion }\end{array}$ & $\begin{array}{c}\text { Right } \\
\text { hand }\end{array}$ & Left hand & Right leg & $\begin{array}{c}\text { Left } \\
\text { leg }\end{array}$ \\
\hline Right hand & $28 / 30$ & $2 / 30$ & $0 / 30$ & $0 / 30$ \\
\hline Left hand & $0 / 30$ & $20 / 30$ & $3 / 30$ & $7 / 30$ \\
\hline Right leg & $0 / 30$ & $3 / 30$ & $23 / 30$ & $4 / 30$ \\
\hline Left leg & $0 / 30$ & $5 / 30$ & $7 / 30$ & $18 / 30$ \\
\hline \multicolumn{5}{|c|}{ SVM } \\
\hline Judgment & $\begin{array}{c}\text { Right } \\
\text { hand }\end{array}$ & Left hand & Right leg & $\begin{array}{c}\text { Left } \\
\text { leg }\end{array}$ \\
\hline Right hand & $26 / 30$ & $4 / 30$ & $0 / 30$ & $0 / 30$ \\
\hline Left hand & $2 / 30$ & $21 / 30$ & $4 / 30$ & $3 / 30$ \\
\hline Right leg & $1 / 30$ & $3 / 30$ & $18 / 30$ & $8 / 30$ \\
\hline Left leg & $1 / 30$ & $1 / 30$ & $3 / 30$ & $25 / 30$ \\
\hline
\end{tabular}

TABLE VI. JUDGMENT RATE OF ALL THE SUBJECTS.

\begin{tabular}{|c|c|c|}
\hline & NN & SVM \\
\hline Subject1 & $86.7 \%$ & $86.7 \%$ \\
\hline Subject2 & $80.0 \%$ & $75.0 \%$ \\
\hline Subject3 & $50.8 \%$ & $58.0 \%$ \\
\hline Subject4 & $74.1 \%$ & $75.0 \%$ \\
\hline Average & $72.9 \%$ & $73.8 \%$ \\
\hline
\end{tabular}

\section{Discrimination Result / Consideration}

Table 2, Table 3, Table 4 and Table 5 show the discrimination results by NN and SVM for each subject. Table 6 shows the discrimination rate for the NN and the SVM. The discrimination rate is a value obtained by dividing the number of times the discrimination for each operation was performed correctly by the number of evaluations.

For subject 1, the discrimination rate of $86.7 \%$ was obtained for both NN and SVM. This was positive discrimination. It was thought that this was a good results even though the study result of discrimination using brain waves at the time of image which were done in the past was considered [1]. However, when comparing with the results of the other subjects, the discrimination rate varied. Since the electrodes and frequency bands used were determined only by the data of subject 1 , it was considered that this was one of the factors that influenced the individual differences. This was because for subject 1 , no change in intensity at rest was observed at all, as shown in Figure 2. On the contrary, when the other three subjects calculate the intensity at rest by the same method, their electrodes showed changes. It was. The difference may have influenced the discrimination results. Therefore, in preprocessing, it may be possible to improve the discrimination rate by examining the optimum frequency band for each subject and finding the electrode and frequency band where the intensity change is not observed at rest.

In addition, when examining erroneous discrimination, subjects 1, 2, and 4 showed a tendency for erroneous discrimination of hand motion was to hand movement and the misidentification of foot motion was to foot movement. However, in Subject 3, in addition to this, there was also a tendency that the misidentification of the right movement tended to be a right motion and the misidentification that the left motion tended to increase the left motion. Therefore, it is possible to discriminate hands and feet to a certain extent, but it was difficult to distinguish between the right and the left.

The averages for the discrimination rates for the $\mathrm{NN}$ and the SVM were equivalent. However, a closer look at the discrimination results for each operation reveals a difference in the discrimination even when using the same feature quantity. Also a difference in classification was found between the NN and the SVM. In general, since SVM has the feature of maximizing margin, it is said to have high general-purpose performance as compared to identification by NN [5]. From this result alone, it is hard to tell which classifier is the best for discriminating brain waves in images. Therefore, it is necessary to investigate further by increasing the number of examinees and using other feature quantities.

Lastly, the parameter setting for the $\mathrm{NN}$ and the SVM was got through trial and error method. More study is required about the influence of parameters settings on the discrimination. The results of user image training should also be considered in the studies.

\section{CONCLUSION}

In this research, we used $\mathrm{NN}$ and SVM to distinguish the four motions of the right hand, the left hand, the right foot and left foot by studying the brain waves during motion imaging. To this end we realized a new BCI system.

For the preprocessing and feature extraction, we investigated the frequency bands of the electrodes and of the brain waves. These were closely related to the motion image processing and the changes in band intensity of brain waves during rest. When the frequency band was limited to $25-30$ $\mathrm{Hz}$, there was no change in intensity at rest. In addition, there was a tendency that the electrode where there was a change in intensity appeared differently with each operation. Therefore the frequency band was limited to $25-30 \mathrm{~Hz}$, and the electrode used the four electrodes $\mathrm{C} 3, \mathrm{C} 4, \mathrm{P} 3$, and P4. This helped to extract the feature amount related to the brain activity during the motion image.

For the motion discrimination, the average of discrimination rates was $72.9 \%$ for $\mathrm{NN}$ and $73.8 \%$ for SVM. 3 out of 4 subjects got a discrimination rate of $70 \%$ or more. 
These results show that it is sufficiently possible to estimate limb movement from brain waves during motion imaging by learning feature quantities extracted from electroencephalogram.

In the future we aim to realize the system in real time in order to treat brain waves as input for interfaces such as auxiliary instruments.

\section{REFERENCES}

[1] Kiyohito Inagaki, Kenji Nakayama, "Classification of mental tasks based on brain waves by neural network", Technical report of the Institute of Electronics, Information and Communication Engineers, Vol. 105, No. 174, pp. 25 - 30, 2005.

[2] Ki-orang Liu, Shin Hibino, Taizo Hanai, Toshiaki Imanishi, Shirataki Tatsuaki, Tetsuo Ogawa, Mitsuaki Matsuhara, Hiroyuki Honda, Takeshi Kobayashi, "Brain wave computer interface Discrimination of right and left elbow flexion images using artificial neural network", Welfare Engineering Symposium Lecture Paper Collection, Vol.2002, No. 2, pp. 137-140, 2002.

[3] Tao Wang, Jie Deng, Bin He, "Classifying EEG-based motor imagery tasks by means of time-frequency synthesized spatial patterns", Clinical Neurophysiology, Vol. 115, No. 12, pp. 2744-2753, 2004.

[4] Eyoshi Sasayama, Tomoyo Iida, Ruku Chung, Takenori Oida, Tetsuo Kobayashi, "Brain information aiming for BMI: Brain wave rhythm analysis during hand movements / images, left / right discrimination", Proceedings of IIP information - intelligence - Precision Equipment Division, Vol.2008, No. 1602, pp. 175-180, 2008.

[5] Hideaki Takai, Kenta Utio, Mitsuaki Kitasaki, Shigeki Nakauchi, "Cognitive state determination by SVM from spontaneous electroencephalogram during task execution", Technical report of the Institute of Electronics, Information and Communication Engineers, Vol.106, No. 407, pp. 25 - 30, 2006.

[6] Hiroki Ichimura, Koichiro Noh, Koichiro Miyamoto, Tatsuo Yoshinobu, "ICA Generation of Spatial Filters to Detect Motion Images from EEG", Technical Report of the Institute of Electronics, Information and Communication Engineers, Vol. 110, No. 295, pp. 53 - 58, 2010.

[7] Minoru Sasaki, Hikari Muto, Harunobu Yasuda, Satoshi Ito, "Estimating Finger Motion Using EEG", Journal of Japan AEM Society, Vol.20, No. 1, pp. 256-261, 2012.

[8] Yoshihiko Kiyoda, Tomohiko Igasaki, Yuuki Hayashida, Nobuyuki Murayama, "EEG analysis at the time of performing the upper limb movement and image", Technical report of the Institute of Electronics, Information and Communication Engineers, Vol.107, No.460, pp. 71- 74, 2008.

[9] Tatsuyuki Kudo, Nobuo Yoshii, "Way of brain waves and way of thinking", Nanzandou, 1966.

[10] Hiroshi Hori, Minoru Shimokawauchi, Nobuhiro Nishiura, Mitsuo Takahashi, Ken Inoue, "Electroencephalogram / Electromyogram Terminology Dictionary", pp. 396-397, Nagai Bookstore, 1991.

[11] Shoichiro Kannoh, Reinhold Scherer, Tatsuo Yoshinobu, Nozomu Hoshiya, Gert Pfurtscheller, "Fundamental Study of BCI System to Detect the Presence or Absence of Images of Foot Action from EEG", Technical Report of the Institute of Electronics, Information and Communication Engineers, Vol. 370, pp. 9 - 12, 2006. 